\title{
O cuidado de enfermagem ao paciente oncológico inserido no ambiente hospitalar
}

\section{durante a pandemia de Covid-19}

\author{
Nursing care for cancer patients inserted in the hospital environment during the Covid-19 \\ pandemic
}

Cuidados de enfermería a pacientes oncológicos insertos en el ambiente hospitalario durante la pandemia de la Covid-19

\author{
Sadi Antonio Pezzi Junior \\ ORCID: https://orcid.org/0000-0001-6606-5112 \\ Universidade Estadual do Ceará, Brasil \\ E-mail: sadi.pezzi@aluno.uece.br \\ Bruna Silva Lima \\ ORCID: https://orcid.org/0000-0001-6122-0013 \\ Universidade Estadual do Ceará, Brasil \\ E-mail: bruninha.lima@aluno.uece.br \\ Ana Claudia de Souza Leite \\ ORCID: https://orcid.org/0000-0003-1407-7634 \\ Universidade Estadual do Ceará, Brasil \\ E-mail: ana.claudia@uece.br \\ Ana Beatriz de Melo Rodrigues \\ ORCID: https://orcid.org/0000-0002-8914-8989 \\ Universidade Estadual do Ceará, Brasil \\ E-mail: biamelo.rodrigues@ aluno.uece.br \\ Raiane Ferreira de Barros \\ ORCID: https://orcid.org/0000-0002-7805-0644 \\ Universidade Estadual do Ceará, Brasil \\ E-mail: raiane.barros@aluno.uece.br \\ Ana Alicia Braz Gomes \\ ORCID: https://orcid.org/0000-0002-4681-5788 \\ Universidade Estadual do Ceará, Brasil \\ E-mail: alicia.braz@aluno.uece.br
}

\begin{abstract}
Resumo
Objetivo: Analisar e sintetizar as evidências científicas disponíveis na literatura sobre a assistência de enfermagem dirigida aos pacientes oncológicos internados em ambiente hospitalar no contexto da pandemia de Covid-19. Metodologia: Revisão integrativa da literatura científica nas seguintes fontes de dados: EMBASE, CINAHL with Full Text, ScienceDirect, Cochrane Clinical Answers, Academic Search Premier, BVS, LILACS, BDENF Enfermagem e PubMed. Os descritores e booleanos usados foram: (Nursing) AND (2019 nCoV Disease) AND (Oncology Care) AND (hospital Care). Foram incluídos seis artigos científicos no estudo. Resultados: Em relação às temáticas identificadas após análise dos estudos, quatro categorias sintetizam os principais aspectos encontrados: organização clínica, dificuldades e percepções de enfermagem no cuidado ao paciente oncológico em contexto hospitalar; o impacto do conhecimento sobre a Covid-19 na prática clínica; estratégias de rastreamento de casos suspeitos; e os cuidados ao paciente oncológico diagnosticado com a doença. Conclusão: É fundamental que, visando a promoção da saúde e prevenção de risco dessa doença, por meio da educação especializada, a enfermagem possa encontrar um caminho favorável, capaz de implementar um manejo eficiente desses pacientes. Evidencia-se que, a partir do levantamento de dados, há escassez de informações acerca da temática. Portanto, torna-se necessário que os profissionais de enfermagem que atuam em ambiente hospitalar, registrem à comunidade científica, informações relacionadas aos desafios presentes na assistência desses pacientes.
\end{abstract}

Palavras-chave: Assistência Hospitalar; Enfermagem; Oncologia; Covid-19.

\footnotetext{
Abstract

Objective: analyze and synthesize from the reading of scientific evidence available in the literature, how nursing care is given to cancer patients who are in a hospital environment in the context of the Covid-19 pandemic. Methodology: Integrative Literature Review based on the methodology proposed by the Joanna Briggs Institute. The following data sources were analyzed: Embase, CINAHL with Full Text, ScienceDirect, Cochrane Clinical Answers, Academic Search
} 
Premier, BVS, LILACS, BDENF Enfermagem and PubMed. The descriptors and Booleans used were: (Nursing) AND (2019 nCoV Disease) AND (Oncology Care) AND (hospital Care). Six scientific articles were included in the study. Results: Regarding the themes identified after analyzing the studies, four categories summarize the main aspects found: clinical organization, difficulties and nursing perceptions in the care of cancer patients in a hospital context; the impact of knowledge about Covid-19 on clinical practice; strategies for tracking suspected cases; and care for cancer patients diagnosed with the disease. Conclusion: It is essential that, in order to promote health and prevent the risk of this disease, through specialized education, nursing can find a favorable path, capable of implementing an efficient management of these patients. It is evident that, from the data collection, there is a lack of information on the subject. Therefore, it is necessary for nursing professionals who work in a hospital environment to provide the scientific community with information related to the challenges present in the care of these patients.

Keywords: Hospital Care; Nursing; Oncology; Covid-19.

\begin{abstract}
Resumen
Objetivo: analizar y sintetizar a partir de la lectura de la evidencia científica disponible en la literatura, cómo se brinda el cuidado de enfermería a los pacientes oncológicos que se encuentran en un ambiente hospitalario en el contexto de la pandemia por Covid-19. Metodología: Revisión integradora de literatura basada en la metodología propuesta por el Instituto Joanna Briggs. Se analizaron las siguientes fuentes de datos: Embase, CINAHL with Full Text, ScienceDirect, Cochrane Clinical Answers, Academic Search Premier, BVS, LILACS, BDENF Enfermagem y PubMed. Los descriptores y booleanos utilizados fueron: (Nursing) AND (2019 nCoV Disease) AND (Oncology Care) AND (hospital Care). Seis artículos científicos fueron incluidos en el estudio. Resultados: En cuanto a los temas identificados después del análisis de los estudios, cuatro categorías resumen los principales aspectos encontrados: organización clínica, dificultades y percepciones de enfermería en el cuidado de pacientes con cáncer en un contexto hospitalario; el impacto del conocimiento sobre Covid-19 en la práctica clínica; estrategias para el seguimiento de casos sospechosos; y atención a pacientes con cáncer diagnosticados con la enfermedad. Conclusión: Es fundamental que, para promover la salud y prevenir el riesgo de esta enfermedad, a través de la educación especializada, la enfermería pueda encontrar un camino favorable, capaz de implementar un manejo eficiente de estos pacientes. Se evidencia que, a partir de la recolección de datos, hay falta de información sobre el tema. Por lo tanto, es necesario que los profesionales de enfermería que actúan en un ambiente hospitalario proporcionen a la comunidad científica información relacionada con los desafíos presentes en el cuidado de estos pacientes.
\end{abstract}

Palabras clave: Atención Hospitalaria; Enfermería; Oncología; Covid-19.

\title{
1. Introdução
}

O novo coronavírus foi caracterizado no dia vinte de março como emergência de saúde pública de importância mundial, classificando-se assim, como pandemia. Segundo a Organização das Nações Unidas (ONU), durante três anos, o Coronavírus disease (Covid-19) ainda está presente em mais de 190 países, dados de janeiro do ano 2022 mostram que os casos notificados ultrapassam 375 milhões, juntamente com a mortalidade em decorrência da doença, equivalente a mais de 5.6 milhões (Who, 2022). A ameaça do Covid-19 está relacionada com algumas características clínicas e epidemiológicas (ainda estando em processo de documentação) e a alta capacidade de transmissibilidade aliado a espectro clínico amplo e letalidade. Diante desse cenário, a possibilidade de infecção tornou-se latente, criando assim, diversos níveis de risco à população (Pavani et al., 2021).

A infecção pelo SARS-CoV-2, vírus respiratório transmitido por contato, gotícula ou aerossol, pode variar de casos assintomáticos, casos leves a manifestações clínicas graves, sendo necessária a atenção aos sinais e sintomas que indicam o quadro clínico do paciente. Os sintomas mais frequentes podem incluir tosse persistente, coriza, febre, desconforto respiratório, até casos críticos que necessitam de suporte respiratório e internações em unidades de terapia intensiva (Brasil, 2021).

Diante do exposto, a partir do risco de infecção por Covid-19, devemos destacar os pacientes oncológicos, que com a presença de imunossupressão secundária ao tratamento ou da imunossupressão relacionada ao adoecimento por câncer, acabam necessitando da atenção das instituições de saúde e por consequência, do cuidado especializado dos profissionais da saúde, principalmente da enfermagem, pois estes também possuem a competência de desenvolver habilidades específicas e fundamentais para o fornecimento do tratamento oncológico adequado em meio a este período (Marchon, Modesto, Rodrigues, Souza \& Plácido, 2020).

Essa doença trouxe grandes desafios para os enfermeiros que estão na linha de frente dos cuidados ao paciente com suspeita ou diagnóstico. Mesmo com as dificuldades apresentadas pelos fatores de superlotação, falta de experiência no manejo 
da doença e falta de equipamentos de proteção individual (EPI's), a enfermagem desempenhou o papel de extrema importância para o enfrentamento desse cenário, adaptando-se, formulando estratégias de cuidado e garantindo uma assistência integral aos pacientes (Lima et al.., 2021).

Neste contexto, os pacientes oncológicos demandam tratamentos contínuos com quimioterapia ou radiografia, os quais são responsáveis por causar baixa resistência imunológica, tendo, pois, grande potencial de serem afetados pela pandemia de Covid-19. Diante disso, os profissionais de enfermagem devem oferecer uma assistência segura e que forneça a continuidade do tratamento, adotando medidas que minimizem os riscos de infecção. A enfermagem atuante no segmento da saúde, é desafiada a flexibilizar, se adaptar às mudanças e a trabalhar em equipe, tendo a perspectiva estreita de desenvolver uma colaboração efetiva de cuidado, levando em conta sua vulnerabilidade diante dessa patologia (Hernandes et al., 2021).

Os pacientes supracitados têm bastante receio de contrair o vírus da Covid-19 ao frequentar hospitais, e ao mesmo tempo, os prestadores de cuidados de saúde na linha de frente correm maior risco de se infectar devido à contínua exposição aos pacientes e seus familiares com pessoas já infectadas por Covid-19. Logo, o treinamento de enfermeiros, o uso das tecnologias digitais, a atuação multiprofissional e a limitação de entrada e saída de familiares podem ser fundamentais para garantir a segurança de todos os envolvidos no ambiente hospitalar (Khosla et al., 2021).

Diante disso, o estudo torna-se relevante por trazer benefícios para a enfermagem, população, pacientes oncológicos e toda a comunidade científica, visando formas de melhorar a qualidade profissional do enfermeiro que trabalha no ambiente hospitalar no contexto da pandemia de Covid-19 e cuida de pacientes oncológicos. Nessa perspectiva, o estudo tem como objetivo analisar e sintetizar a partir da leitura de evidências científicas disponíveis na literatura, como se dá a assistência de enfermagem dirigida aos pacientes oncológicos que estão em ambiente hospitalar no contexto da pandemia de Covid-19.

\section{Metodologia}

Estudo do tipo revisão integrativa da literatura que sintetiza os conhecimentos e a incorporação da aplicabilidade de resultados de estudos significativos na prática (Souza, Silva \& Carvalho, 2010). Foi desenvolvido em dezembro de 2021, segundo as recomendações do Instituto Joanna Briggs (JBI) a partir da preferência dos autores pela produção e apresentação crítica do material já publicado (Pearson et al., 2005). Foram adotados e elaborados seis etapas para a formulação e organização do Preferred Reporting Items for Systematic reviews and Meta-Analyses extension for Scoping Reviews (PRISMA-ScR), sendo eles: identificação da questão norteadora; estabelecimento de critérios para inclusão e exclusão dos estudos; definição das informações a serem extraídas dos estudos selecionados; avaliação dos estudos incluídos; interpretação dos resultados e apresentação da revisão (Galvão et al., 2015).

As definições de População, Interesse, Comparação e Contexto (PICO) foram utilizadas como estratégia para guiar a coleta de dados e classificar a qualidade dos resultados. Com essa perspectiva, definiu-se: $\mathrm{P}=$ Pacientes Oncológicos; I= Assistência de enfermagem; $\mathrm{C}=$ não houve comparação; e $\mathrm{O}=$ Pandemia de Covid-19 (Santos et al., 2007). Esses três componentes foram os elementos fundamentais para formulação da questão de pesquisa, juntamente com a estratégia de delimitação de busca bibliográfica de evidências. Desse modo, a pergunta da pesquisa formulada foi: Quais as evidências científicas acerca da assistência de enfermagem aos pacientes oncológicos em ambiente hospitalar no contexto da pandemia de Covid-19?

A busca por informações foi realizada no Portal de Periódicos da Capes/MEC, com área de conhecimento sendo ciências da saúde e subáreas sendo enfermagem, nas seguintes bases de dados: Embase (Elsevier), CINAHL with Full Text (EBSCO), ScienceDirect (Elsevier), Cochrane Clinical Answers - CCA, Academic Search Premier. Também, na Biblioteca Virtual em Saúde (BVS) nas bases: Literatura Latino-Americana e do Caribe em Ciências da Saúde (LILACS) e BDENF Enfermagem e na Biblioteca Nacional de Medicina (PubMed/Medline). 
Os descritores em Ciências da Saúde (DeCS/MeSH) foram selecionados e validados após teste e re-teste, encontrandose em português e em inglês: Enfermagem, Covid-19, Oncologia e Assistência Hospitalar e Nursing, 2019 nCoV Disease, Oncology Care e Hospital Care. Em seguida, empregou-se estratégia de busca com combinação de operadores booleanos para a seleção das evidências científicas da seguinte forma: (Nursing) AND (2019 nCoV Disease) AND (Oncology Care) AND (Hospital Care), em inglês. Além disso, também foi empregado a combinação (Enfermagem) E (Covid-19) E (oncologia) E (Assistência Hospitalar), em português.

Os critérios de inclusão foram: artigos científicos, publicados no período de 2020 a 2021, disponibilizados por revistas nacionais e internacionais, contendo texto completo e acesso livre. Foram selecionados por título, resumo e descritores aqueles que respondessem à pergunta norteadora do estudo e possuíam, no mínimo, os quatro maiores níveis de evidências científicas. Todos os idiomas foram incluídos. Já os critérios de exclusão, foram: artigos de literatura cinza, revisões integrativas e duplicações.

Para a verificação dos níveis de evidências (NE) dos artigos selecionados para o estudo, foi utilizada a Classificação de Oxford Centre for Evidence-Based Medicine (2009), delimitando a partir de estudos de nível "1a" a "5" o entendimento de que as revisões sistemáticas de ensaios clínicos controlados e randomizados possuem maior nível, ou seja, nível 1 de evidência. Já o nível 5, sendo o menor nível, contém opinião desprovida de avaliação crítica ou baseada em matérias básicas.

A análise dos estudos ocorreu em pares, por meio da leitura e releitura da amostra com a confecção de fichamentos na ferramenta Excel®, um editor de planilhas produzido pela Microsoft@. Esses resultados foram apresentados por quadros analíticos, sendo utilizada a letra inicial "A" para representar artigo e um número seguindo sequência conforme foi incluído, facilitando assim a compreensão e organização dos resultados selecionados. Por fim, realizou-se a interpretação do material empírico através dos quadros analíticos com as informações pertinentes de cada estudo.

\section{Resultados e Discussão}

Na etapa de identificação, para compor o corpo dos nossos resultados, foram levantados (491) artigos. Destes, apenas (74) foram identificados através da leitura de título, sendo (7) excluídos por estarem duplicados. Na etapa de seleção, após a leitura dos títulos e resumos, (67) foram escolhidos. Destes, apenas (17) passaram para a fase de elegibilidade, pois (50) não atendiam à pergunta problema. Na etapa de elegibilidade, após a análise do $1^{\circ}$ revisor, direcionamos os (17) estudos para o $2^{\circ}$ revisor e este, conforme os critérios de inclusão e exclusão, determinou que apenas (6) atendiam minimamente aos nossos critérios. Logo, os (6) estudos restantes passaram para a fase de inclusão, sendo utilizados na composição dos resultados deste artigo. As etapas da seleção estão indicadas no fluxograma abaixo (Figura 1), sendo de finalidade organizacional, garantindo um bom entendimento da nossa seleção. 
Figura 1 - Fluxograma de identificação dos estudos incluídos e excluídos.

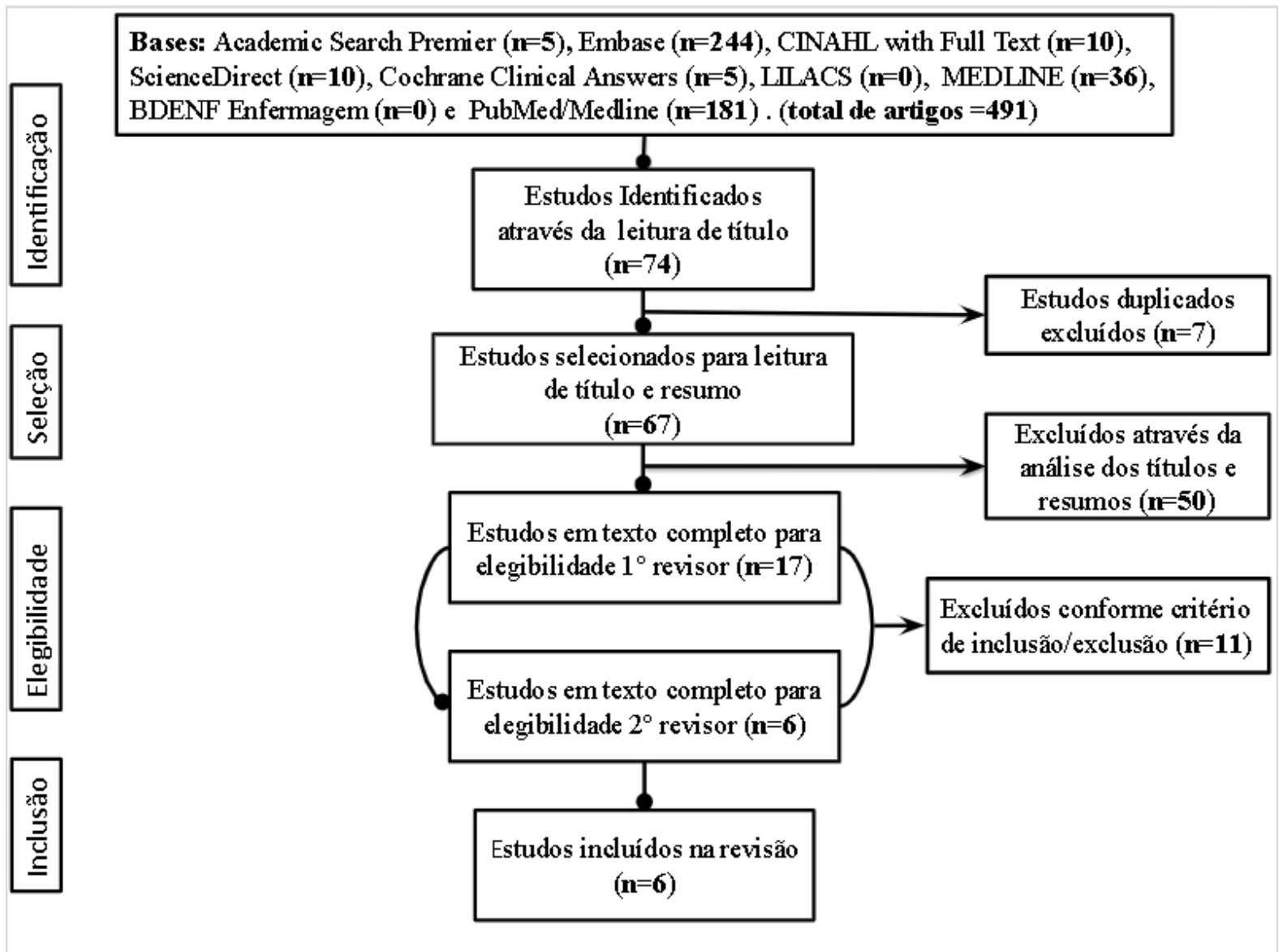

Fonte: Autores (2022).

Em relação aos Níveis de Evidências (NE), foram identificados (4) estudos NE 3b e (2) NE 4. Também, os artigos identificados no estudo foram desenvolvidos em quatro países, sendo eles: Bélgica e Itália, com dois artigos, respectivamente; Turquia e Brasil com um artigo cada. É importante destacar ainda que os idiomas identificados foram inglês (5) e português (1), além disso, os estudos identificados foram publicados no ano de 2020 (2) e 2021 (4). As características dos estudos encontrados estão descritas abaixo (Quadro 1). 
Quadro 1 - Contendo as características dos estudos encontrados de acordo com Autor/Ano, País, Tipo de estudo/Níveis de Evidência (NE), População/Amostra e Objetivos.

\begin{tabular}{|c|c|c|c|c|}
\hline Autor/Ano & País & $\begin{array}{c}\text { Tipo de } \\
\text { Estudo/NE }\end{array}$ & População/Amostra & Objetivos \\
\hline $\begin{array}{l}\text { Ballatore et al./ } \\
\qquad(2021)\end{array}$ & Itália & $3 b$ & $\begin{array}{l}383 \text { profissionais de saúde } \\
\text { oncológicos }\end{array}$ & $\begin{array}{c}\text { Documentar o estado atual da } \\
\text { oncologia italiana e avaliar as } \\
\text { dificuldades que surgiram no manejo } \\
\text { de pacientes com câncer durante a } \\
\text { pandemia da SARS-Cov- } 2 \text {. }\end{array}$ \\
\hline $\begin{array}{l}\text { Sampaio et al./ } \\
\text { (2021) }\end{array}$ & Bélgica & $3 b$ & $\begin{array}{l}327 \text { pacientes admitidos pelo } \\
\text { Departamento de Emergência } \\
\text { (DE) da Unidade de Terapia } \\
\text { Oncológica Paliativa (UTOP) }\end{array}$ & $\begin{array}{c}\text { Avaliar a eficiência de critérios } \\
\text { clínicos adotados para identificar } \\
\text { casos suspeitos de Covid-19 pelo DE } \\
\text { da UTOP. }\end{array}$ \\
\hline $\begin{array}{l}\text { Santiago/ } \\
(2021)\end{array}$ & Brasil & 4 & $\begin{array}{c}\text { Enfermeiros que vivenciaram a } \\
\text { experiência acerca da assistência } \\
\text { ao paciente com } \\
\text { Covid-19 em uma instituição de } \\
\text { referência. }\end{array}$ & $\begin{array}{l}\text { Relatar a experiência da assistência } \\
\text { de Enfermagem à primeira paciente } \\
\text { em cuidados paliativos oncológicos } \\
\text { com Covid-19, no Rio de Janeiro. }\end{array}$ \\
\hline $\begin{array}{c}\text { Semerci et al./ } \\
(2020)\end{array}$ & Turquia & $3 b$ & 185 enfermeiros oncologistas & $\begin{array}{c}\text { Avaliar o conhecimento dos } \\
\text { enfermeiros de oncologia turcos } \\
\text { sobre Covid-19 durante o surto atual } \\
\text { na Turquia. }\end{array}$ \\
\hline $\begin{array}{c}\text { Van Dam et al./ } \\
\text { (2021) }\end{array}$ & Bélgica & $3 b$ & $\begin{array}{l}922 \text { pacientes com câncer, } 100 \\
\text { pacientes sem câncer e } 94 \\
\text { profissionais de saúde }\end{array}$ & $\begin{array}{l}\text { Documentar a prevalência de Covid- } \\
19 \text { e soroprevalência de SARS-CoV- } \\
2 \text { IgG / Ig total em pacientes com } \\
\text { câncer, e pacientes sem câncer } \\
\text { frequentando a Unidade de } \\
\text { Oncologia Multidisciplinar de um } \\
\text { hospital. }\end{array}$ \\
\hline $\begin{array}{l}\text { Zeneli et al./ } \\
\qquad(2020)\end{array}$ & Itália & 4 & $\begin{array}{l}\text { Pacientes com câncer de todos os } \\
\text { ambientes de cuidados, incluindo } \\
\text { pacientes } \\
\text { em acompanhamento que } \\
\text { tiveram Covid-19 confirmado. }\end{array}$ & $\begin{array}{l}\text { Descrever a resposta à emergência } \\
\text { do Covid-19 em um centro de câncer } \\
\text { para permitir que outras organizações } \\
\text { de enfermagem determinem } \\
\text { elementos que podem ser úteis para } \\
\text { gerenciar um surto de pacientes em } \\
\text { seu próprio ambiente. }\end{array}$ \\
\hline
\end{tabular}

Fonte: Autores (2022).

Em relação às temáticas identificadas após análise dos estudos, quatro categorias sintetizam os principais aspectos encontrados sobre a assistência de enfermagem aos pacientes oncológicos inseridos em ambiente hospitalar no contexto da pandemia de Covid-19: organização clínica para correção do distanciamento social e para a prevenção da propagação do vírus, dificuldades e percepções; o impacto do conhecimento sobre a Covid-19 na prática clínica; estratégias de rastreamento de casos suspeitos; e os cuidados ao paciente oncológico diagnosticado com a doença.

O advento da pandemia de Covid-19 gerou um impacto para os profissionais da área de oncologia e para os demais profissionais da saúde. Tendo em vista o cenário da pandemia, foi necessário reorganizar a assistência e a abordagem dos pacientes oncológicos, pois estes encontram-se mais suscetíveis à contaminação por Covid-19. A necessidade da reorganização clínica e a identificação das novas formas de manejo com os pacientes trouxe à tona mais uma preocupação para os profissionais da oncologia, que sentem medo e ansiedade em relação ao adiamento dos tratamentos, que acabam tornando-se qualitativamente inferiores ao período pré-pandemia (Ballatore et al., 2021). 
Conforme Ramos (2020), para que a manutenção do tratamento oncológico não se torne um risco frente à possibilidade de adquirir Covid-19, o enfermeiro deve realizar uma avaliação individualizada do paciente, seguindo protocolos e também orientando as medidas de proteção como higiene das mãos e o uso de máscaras. Além disso, o profissional supracitado também pode atuar na reorganização dos espaços de atendimento, visto que existe a possibilidade de contato de pacientes oncológicos com pacientes possivelmente infectados.

De acordo com Zeneli et al. (2020) a partir do planejamento do fluxo de trabalho de enfermagem tem-se a aquisição de novas habilidades e conhecimentos, estratégias de comunicação eficazes, controle de infecção, políticas, programas de avaliação e vigilância de riscos e fornecimento contínuo de EPI's, adotando essas estratégias a equipe de enfermagem é capaz de melhorar o distanciamento social e de prevenir que pacientes oncológicos e a própria enfermagem reduzam as possibilidades de infecção pelo vírus da Covid-19 (Zeneli et al., 2020).

Ademais, em relação aos profissionais de saúde, nota-se uma sobrecarga de estresse maior. No estudo de Ballatore et al. (2021), a principal consequência psicológica foi a piora na qualidade do sono, o que afetou tanto o humor, quanto a concentração. Logo, pode-se observar que estes profissionais se encontram mais suscetíveis a desenvolver sintomas de estresse e ansiedade, sendo necessário fornecer apoio e tratamento de saúde mental para estes trabalhadores.

Para Semerci et al. (2021), a pandemia de Covid-19 gerou desafios significativos para os profissionais de saúde em todas as áreas, particularmente centros de câncer. Durante a pandemia, especialmente os enfermeiros devem ter conhecimento sobre a Covid-19 e, assim, agir de forma a prevenir a transmissão e detectar os primeiros sinais de infecção entre pacientes com câncer. Os enfermeiros oncológicos desempenham um papel importante na gestão e prevenção da propagação do SARS-CoV-2, assim como na educação em saúde do paciente. Dessa forma, a realização de treinamentos, testes de conhecimentos e produção de materiais informativos são fundamentais.

Além disso, o estudo de Oliveira, Migueis, Silva \& Oliveira (2021) afirma que as lideranças de saúde, a administração de enfermagem e a gestão hospitalar devem apoiar os enfermeiros com uma formação abrangente, currículo composto por uma abordagem mais estruturada para fornecer conscientização profissional suficiente sobre a Covid-19. É importante destacar a necessidade de pesquisas que relatam a realidade dos profissionais e o nível do conhecimento diante de uma doença nova, altamente mutável e com várias variantes identificadas. A produção de trabalhos que abordem a Covid-19 e suas particularidades são indispensáveis, buscando a atualização e qualidade de assistência prestada.

O novo cenário da pandemia de Covid-19 trouxe grandes desafios para a assistência aos pacientes oncológicos. Devido a isto, estratégias para garantir atendimento foram testadas. O diagnóstico rápido e confiável dos casos suspeitos de Covid-19 em pacientes oncológicos foi o critério clínico utilizado no departamento de uma unidade de atendimento de oncologia paliativa de referência nacional, e se mostrou bastante eficiente. Destaca-se, portanto, como um importante elemento para a implementação de estratégias voltadas para a melhoria da assistência aos pacientes oncológicos durante a pandemia causada pelo coronavírus. O cuidado de qualidade deve começar com o correto diagnóstico dos casos (Sampaio et al., 2021).

Ademais, para Magno et al. (2020), a combinação de estratégias com a ampla testagem apresentou melhoras no controle da pandemia em alguns países da Ásia e Oceania. Em Singapura a testagem de todos os casos suspeitos e dos contactantes contribuiu para a deteç̧ão precoce de aproximadamente metade dos casos de Covid-19, além de ter sido uma estratégia efetiva para a redução progressiva do surgimento de novos casos da doença no início da pandemia. Esse retorno positivo do diagnóstico precoce, mostra a importância da ampliação das testagens.

No contexto da pandemia de SARS-CoV-2, compreender a taxa de infecção assintomática e imunidade pré-existente é fundamental para avaliar o impacto da propagação pandémica do coronavírus na comunidade de pacientes com câncer. No entanto, estimativas sobre a soro prevalência de SARS-CoV-2 em populações de câncer dependem fortemente do tipo de teste sorológico utilizado, a população testada e tempo de teste, particularmente relacionados com a extensão da pandemia do 
coronavírus na população geral do local. Uma pesquisa nas unidades mostrou que pacientes com câncer se protegiam muito bem contra SARS-CoV-2 por isolamento social, uso de máscaras faciais e higiene das mãos. Isso se soma às medidas que foram tomadas nos hospitais para fornecer cuidados oncológicos de forma mais segura possível (Van Dam et al., 2021).

Além disso, o estudo de Xavier et al. (2020) relata que devido a pandemia do coronavírus ser um evento recente, os testes imunológicos ainda se encontram em processo de desenvolvimento e validação, para estarem aptos para a inserção como uma ferramenta diagnóstica confiável e descomplicada de usar no novo cenário pandêmico. Os fatores individuais de cada ser humano refletem na resposta imunológica dos testes, o resultado depende do paciente e das características do antígeno utilizado, e dependendo do paciente o aumento de anticorpos pode ser precoce ou tardio. Estudos científicos discutem que a resposta imune ao novo coronavírus ainda é muito limitada.

O plano de cuidados da equipe de enfermagem ao paciente oncológico em estágio de doença avançada e acometido pela Covid-19 se torna bastante específico, múltiplo e complexo, devido à rápida progressão no curso dessa enfermidade, demandando cuidados físicos, psíquicos e socioculturais. A piora do quadro clínico em curto período, faz com que a assistência de enfermagem se volte à necessidade de desenvolver um cuidado integral e humanizado, onde o enfermeiro compreenda a necessidade desse paciente, criando vínculo de empatia e satisfazendo as verdadeiras necessidades. O isolamento faz o paciente se sentir vulnerável e solitário, em caso de morte, o enfermeiro deve permanecer ao seu lado, sendo a companhia que conforta, de forma a estabelecer o cuidado até o fim (Santiago \& Silva, 2020).

Dessa forma, Nascimento et al. (2021) afirma que com base nos aspectos do tratamento oncológico e nas mudanças acarretadas pela pandemia da Covid-19, faz-se necessária a elaboração de estratégias para amenizar o impacto desse período no bem-estar, diagnóstico, prognóstico e tratamento dos pacientes com câncer. No decorrer do processo de quimioterapia o paciente recebe cuidados integrais e multiprofissionais assegurando sua dignidade de tratamento, assim como a espera de resultados satisfatórios. Dessa maneira, o enfermeiro é o profissional que mais dedica tempo e assistência a esse grupo, tornando-se um protagonista nos cuidados, onde o paciente e seus familiares conferem maior confiança para o encorajamento na realização do tratamento.

\section{Conclusão}

O contexto da pandemia de Covid-19 trouxe grandes desafios para a enfermagem que atua em ambiente hospitalar, esta que vêm trabalhando no segmento da saúde e sendo desafiada a flexibilizar, a se adaptar às mudanças e a desenvolver novas estratégias de cuidados. É fundamental que, visando a promoção da saúde e prevenção de risco dessa doença, por meio da educação especializada, a enfermagem possa encontrar um caminho favorável capaz de implementar um manejo eficiente de pacientes oncológicos.

Apesar disso, evidencia-se que, a partir do levantamento de dados, há escassez de informações acerca da temática. Portanto, torna-se necessário que os profissionais de enfermagem que atuam em ambiente hospitalar no contexto da Covid-19, registrem à comunidade científica, informações relacionadas a assistência, prática clínica, adaptação e desafios presentes na assistência aos pacientes supracitados, tendo em vista a importância do acesso desse tipo de informação para a formulação de novos estudos e principalmente, para a formação de profissionais mais qualificados nos cuidados a pacientes oncológicos.

\section{Referências}

Ballatore, Z., Bastianelli, L., Merloni, F., Ranallo, N., Cantini, L., Marcantognini, G., \& Berardi, R. (2020). Scientia Potentia Est: how the Italian world of oncology changes in the COVID-19 pandemic. JCO Global Oncology, 6, 1017-1023.

Brasil. (2021). Ministério da Saúde, Secretaria de Vigilância em Saúde. Guia de vigilância epidemiológica Emergência de saúde pública de Importância nacional pela Doença pelo coronavírus $2019-$ covid-19. https://www.conasems.org.br/wp-content/uploads/2021/03/Guia-de-vigila\%CC\%82nciaepidemiolo\%CC\%81gica-da-covid_19_15.03_2021.pdf 
Centre for Evidence-based Medicine. (2009). Oxford Center for Evidence-based Medicine: levels of evidence. https://www.cebm.ox.ac.uk/resources/levels-ofevidence/oxford-centre-for-evidence-based-medicine-levels-of-evidence-march-2009

Galvão, T. F., Pansani, T. S. A., \& Harrad, D. (2015). Principais itens para relatar Revisões sistemáticas e Meta-análises: a recomendação PRISMA. Rev. Epidemiol. Serv. Saúde, 24, 335-342.

Hernandes, L. O., Vitorino P. G. S, Mejía, J. V .C., Lima, T. O. P., Chã, N. V., Flauzino, V. H. P., Cusato, T. V., Gomes, D. M., Ribeiro, D. V., \& Cesário, J. M. S. (2021). Cuidados de enfermagem ao paciente oncológico acometido pela Covid-19. Rev. Soc. Dev, 10 (9), e26410918099-e26410918099, 1-10.

Khosla, D., Zaheer, S., Madan, R., Goyal, S., Kumar, N., \& Kapoor, R. (2021). Tele-oncology in cancer care during COVID-19 pandemic-expanding role in the developing world. Clin. Cancer Investig. J, 10 (4), 182-185.

Lima, B. S., Barros, R. F., Gomes, A. A. B., Pezzi Junior, S. A., Rodrigues, A. B. M., \& Leite, A. C. S. (2021). Atuação de enfermagem diante da Pandemia da COVID-19 a nível hospitalar. Braz. J. Health Rev, 4 (6), 26339-26352.

Magno, L., Rossi, T. A., Mendonça-Lima, F. W., Santos, C. C., Campos, G. B., Marques, L. M.., \& Dourado, I. (2020). Desafios e propostas para ampliação da testagem e diagnóstico para COVID-19 no Brasil. Cienc. Saude colet, 25, 3355-3364.

Marchon, R. M., Modesto, F. C., Rodrigues, C. C. L., Souza, P. L., \& Plácido, T. R. (2020). Cuidados da fisioterapia no paciente oncológico com Covid-19. Rev. Bras. Cancerol, 66, 1-4.

Mendes, K. D. S., Silveira, R. C. C. P., \& Galvão, C. M. (2008). Revisão integrativa: método de pesquisa para a incorporação de evidências na saúde e na enfermagem. Texto contexto-enferm, 17 (4),758-764.

Nascimento, D. T., Koeppe, G. B. O., Oliveira, P. P., Valadão, R. R., Prado, T. S. B., Santos, N. S., \& Cerqueira, L. C. N. (2021). Estratégias de saúde para manutenção da qualidade da assistência na quimioterapia no contexto da pandemia da COVID-19. Glob. Acad. Nurs. Journ., 2, e117, 1-10.

Oliveira, W. S., Migueis, G. S., Silva, M. S., \& Oliveira, W. J. (2021). Conhecimento sobre Covid-19 dos profissionais de enfermagem atuantes no enfrentamento da doença. Rev. Soc. Dev, 10 (11), e244101119676-e244101119676, 1-13.

Pavani, F. M., Silva, A. B. S., Olschowsky, A., Wetzel, C., Nunes, C. K., \& Souza, L. B. (2021). Covid-19 and repercussions in mental health: a narrative review of literature. Rev. gaúcha de enferm., 42, e20200188, 1-14.

Pearson, A., Wiechula, R., Court, A., \& Lockwood, C. (2005). The JBI model of evidence-based healthcare. Int. J. Evid. Based Healthc., 3 (8), $207-215$.

Pellizzon, R. F. (2004). Pesquisa na área da saúde: 1. Base de dados DeCS (Descritores em Ciências da Saúde). Acta Cirúrgica Brasileira, 19 , $153-163$.

Ramos, R. S. (2020). A Enfermagem Oncológica no enfrentamento da pandemia de Covid-19: reflexões e recomendações para a prática de cuidado em oncologia. Rev. Bras. de Cancerol., 66, e-1007, p. 1-5.

Sampaio, S. G. S. M., Dias, A. M., Freitas, R., Borsatto, A. Z., Esteves, E. M. F. L., \& Oliveira, L. C. (2021). Evaluation of the criteria adopted to identify suspected cases of COVID-19 in the emergency department service of a Referral palliative oncology care unit. Am. J. Hosp. Palliat. Care, 38 (2), $199-203$.

Santos, C. M. C., Pimenta, C. A. M., \& Nobre, M. R. C. (2007). A estratégia PICO para a construção da pergunta de pesquisa e busca de evidências. Rev. Lat.Am. Enferm, 15 (3), 508-511.

Santiago, F. B., \& Silva, A. L. A. (2020). Primeiro caso de COVID-19 em uma unidade de Cuidados Paliativos oncológicos: relato de experiência. Enferm. em Foco, 11 (2. ESP). 205-210.

Semerci, R., Kudubes, A. A., \& Esref, F. Ç. (2021). Assessment of Turkish oncology nurses' knowledge regarding COVID-19 during the current outbreak in Turkey. Support Care Cancer, 29 (4), 1999-2006.

Souza, M. T., Silva, M. D., \& Carvalho, R. (2010). Revisão integrativa: o que é e como fazer. einstein, 8 (1), $102-106$.

Van Dam, P., Huizing, M., Roelant, E., Hotterbeekx, A., Winter, F. H., Kumar-Singh, S., Moons, P., Amajoud, Z., Vulsteke, C., Croes, L., Janssens, A., Berneman, Z., Prenen, H., Meuris, L., Berghe, W. V., Smits, E., \& Peeters, M. (2021). Immunoglobin G/total antibody testing for SARS-CoV-2: a prospective cohort study of ambulatory patients and health care workers in two Belgian oncology units comparing three commercial tests. Eur. J. Cancer, 148, 328-339.

World Health Organization. (2022). Painel da OMS sobre o coronavírus (COVID-19). https://covid19.who.int/

Xavier, A. R., Silva, J. S., Almeida, J. P. C. L., Conceição, J. F. F., Lacerda, G. S., \& Kanaan, S. (2020). COVID-19: manifestações clínicas e laboratoriais na infecção pelo novo coronavírus. J. Bras. Patol. Med. Lab, 56, 1-9.

Zeneli, A., Altini, M., Bragagni, M., Gentili, N., Prati, S., Golinucci, M., Rustignoli, M., \& Montalti, S. (2020). Mitigating strategies and nursing response for cancer care management during the COVID-19 pandemic: an Italian experience. Int Nurs Rev, 67 (4), 543-553. 